\title{
Mott insulator-superfluid transitions in a two-band model at finite temperature and possible application to supersolid ${ }^{4} \mathrm{He}$
}

\author{
Huai-Bin Zhuang, ${ }^{1}$ Michael Ma, ${ }^{2} \mathrm{Xi}$ Dai, ${ }^{1}$ and Fu-Chun $\mathrm{Zhang}^{1}$ \\ ${ }^{1}$ Centre of Theoretical and Computational Physics and Department of Physics, The University of Hong Kong, Hong Kong \\ ${ }^{2}$ Department of Physics, University of Cincinnati, Cincinnati, Ohio 45221, USA \\ (Received 28 April 2007; revised manuscript received 8 August 2007; published 7 November 2007)
}

\begin{abstract}
We study the Mott insulator-superfluid transition in a two-band boson Hubbard model, which can be mapped onto a spin-1/2 XY model with spins coupled to an additional Ising degree of freedom. By using a modified mean field theory that includes the effects of phase fluctuations, we show that the transition is first order at both zero and finite temperatures. On the Mott insulator side, there may be reentrance in phase transition. These features are consequences of the underlying transition between competing defect poor and defect rich phases. The possible relevance of the model and our results to supersolid ${ }^{4} \mathrm{He}$ and cold bosonic atoms in optical lattices are discussed.
\end{abstract}

DOI: $10.1103 /$ PhysRevB.76.174504

PACS number(s): 67.80.-s, 03.75.Hh, 05.30.-d, 67.40.-w

\section{INTRODUCTION}

The Mott insulator-superfluid (MI-SF) transition is one of the striking phenomena arising from the many body physics of correlated boson systems on a lattice. ${ }^{1}$ The physics of this transition is believed to be relevant to not only systems with bosons as "elementary particles," but also condensed matter systems whose low energy physics is governed by bosonic degrees of freedom, for example, an array of quantum Josephson junctions. Recently, there has been a renewed interest in the MI-SF transition due to its relevance to two types of experimental systems. The first is supersolidity of solid ${ }^{4} \mathrm{He}$ (Refs. 2 and 3) if one views the normal solid to supersolid transition as a Bose condensation transition that occurs due to delocalization of the ${ }^{4} \mathrm{He}$ atoms without melting the underlying crystalline lattice. The second is ultracold bosonic atoms in an optical lattice. ${ }^{4}$

The commonly accepted paradigm for the MI-SF transition is based on the one-band boson Hubbard model. In that model, the MI-SF transition is a continuous one with the Mott-Hubbard gap and the condensate density increasing continuously from 0 on the MI and the SF sides of the transition, respectively. Recently, Dai, Ma, and Zhang (DMZ) ${ }^{5}$ proposed a two-band Hubbard Hamiltonian as a model for solid ${ }^{4} \mathrm{He}$, and showed using a single-site mean field theory that the MI-supersolid transition can be first order. In this paper, we seek to investigate the validity of this claim beyond mean field theory by including the effects of phase fluctuations of the condensate. Specifically, we consider the following spin $S=\frac{1}{2} X Y$ model on a lattice, where the spins are coupled to an additional Ising degree of freedom

$$
H=\Delta \sum_{i} \hat{n}_{i}-h \sum_{i} \hat{S}_{i}^{z} \hat{n}_{i}-J \sum_{\langle i j\rangle}\left(\hat{S}_{i}^{+} \hat{S}_{j}^{-}+\hat{S}_{i}^{-} \hat{S}_{j}^{+}\right) \hat{n}_{i} \hat{n}_{j} .
$$

Here the Ising variable $\hat{n}_{i}=0,1$ and $\hat{S}_{i}^{+}$and $\hat{S}_{i}^{-}$are the raising and lowering operators. As a model of magnetism, this Hamiltonian would describe a $X Y$ spin system in a transverse magnetic field $h$, with $n_{i}=0,1$ representing the absence (presence) of a spin on site $i$. In this case $\Delta$ plays the role of a chemical potential for the spin. For example, $H$ can act as a model for an electronic insulator with two on-site elec- tronic configurations, one magnetic and one nonmagnetic separated by energy $\Delta$, or it can describe a binary alloy of magnetic and nonmagnetic ions. However, for this work, we are more interested in $H$ as a model of boson insulatorsuperfluid transition. Below we will discuss the relevance of this model to the boson two-band Hubbard model and to supersolid ${ }^{4} \mathrm{He}$ and trapped bosons in an optical lattice.

\section{A. Supersolid ${ }^{4} \mathrm{He}$}

The possibility of supersolidity in ${ }^{4} \mathrm{He}$, which refers to the coexistence of crystalline ordering and superfluidity, was proposed some years ago by Andreev and Lifshitz, ${ }^{6}$ Chester, ${ }^{7}$ and Leggett. ${ }^{8}$ The interest in such a unique state of matter is recently rekindled following the report by Kim and Chan ${ }^{2,3}$ of the observation of nonclassical rotational inertia (NCRI) in solid ${ }^{4} \mathrm{He}$ confined inside porous media and in bulk solid ${ }^{4} \mathrm{He}$ at low temperature. NCRI has since been confirmed by other groups, ${ }^{9-11}$ although it is still debatable as to whether this is a bulk equilibrium effect or a result of nonequilibrium defects. ${ }^{9} 12$ Direct numerical calculations strongly indicate that off-diagonal long ranged order (ODLRO) is not present at equilibrium in bulk solid ${ }^{4} \mathrm{He} .{ }^{13}$ Furthermore, experiments have failed to detect direct superflow or "superconductivity" 14,15 unless grain boundaries are seen to be present. ${ }^{15}$ This leads to grain boundaries as a natural explanation of the observation of NCRI. ${ }^{16}$ On the other hand, recently Clark et al. ${ }^{17}$ reported the observation of NCRI in single crystal helium. Therefore, it remains controversial as to whether bulk ${ }^{4} \mathrm{He}$ is a supersolid at low temperature. In our opinion, these recent experiments provide the impetus for a better theoretical understanding of possible mechanisms for bulk equilibrium supersolidity. In addition, independent of solid ${ }^{4} \mathrm{He}$, the question of equilibrium supersolidity in quantum crystals is an issue of fundamental interest. In their seminal paper, Andreev and Lifshitz ${ }^{6}$ proposed that since ${ }^{4} \mathrm{He}$ is a quantum solid with large zero point motion, a finite density of defects may be present even in the ground state. Amongst these defects, the most promising candidate is zero point vacancies, whose motion they argued will be wavelike at low temperature and so will Bose con- 
dense. The Bose condensation of the vacancies can then lead to superfluidity without destroying the underlying crystalline ordering. From the point of view of the presence of ODLRO in Jastrow wave functions describing solids, $\mathrm{Chester}^{7}$ also conjectured zero point vacancies as the mechanism for supersolidity. If vacancies are present, the solid will be incommensurate, with the number of $\mathrm{He}$ atoms different from the number of lattice sites. Recently, Anderson et al. ${ }^{18}$ pointed to the $T^{7}$ correction to the specific heat as evidence of incommensurability in the ground state.

While appealing, the idea of zero point vacancies is subject to stringent constraint from both experiments and several computational calculations, showing vacancies as activated from the commensurate solid. The activation energy is found to be about $10 \mathrm{~K}$ from $\mathrm{x}$-ray diffraction ${ }^{19}$ and about $15 \mathrm{~K}$ in simulations. ${ }^{13}$ This is at odds with the Andreev and Lifshitz's proposal that the commensurate ground state is unstable with respect to spontaneous generation of vacancies. Recently, DMZ $^{5}$ proposed a possible solution to the quandary by including a lower energy defect that they called the exciton, which is an on-site bound state of a vacancy and an interstitial. Physically, this defect corresponds to a broadening distortion of the local wave function. For example, if the defectfree solid state is given by the (unsymmetrized) HartreeNosanow wave function ${ }^{20}$

$$
\Psi_{0}=\prod_{i} \phi_{a}\left(r_{i}-R_{i}\right),
$$

where $\phi_{a}$ is a localized wave function, then the state with one exciton defect on the site $j$ can be written as

$$
\Psi_{0}=\phi_{b}\left(r_{j}-R_{j}\right) \prod_{i \neq j} \phi_{a}\left(r_{i}-R_{i}\right),
$$

where $\phi_{b}$ is also localized, but less so than $\phi_{a}$. The key idea is that an atom in the state $\phi_{b}$ can tunnel more effectively into a neighboring vacancy site (and vice versa) than one in the state $\phi_{a}$ due to the wave function broadening. As a result, even though vacancies are activated in a defect-free background, they can be spontaneously generated in an exciton background. At low temperature, they will then Bose condense and the condensation energy may overcome the energy cost of creating exciton defects. In their theory, the commensurate solid would then be a metastable state, while the true ground state will be an incommensurate one with finite densities of vacancies and excitons. The metastability of the commensurate solid has also been proposed by Anderson et $a l .{ }^{18}$ Although the numerical calculation of Boninsegni et al. found the commensurate solid to be stable, ${ }^{21}$ it has been questioned whether that result is due to finite-sized effects. ${ }^{22}$ On the experimental front, recent neutron scattering measurements found no discernible change in commensurability between the normal solid and supersolid. ${ }^{23}$

As a model for their theory, DMZ considered a two-band Hubbard model, in which the defect-free (DF) state is considered the "vacuum," and the boson operators are operators that create and destroy defects. In this paper, it is more convenient to take the vacuum as the physical vacuum for ${ }^{4} \mathrm{He}$ atoms, in which case the DMZ Hamiltonian becomes

$$
\begin{aligned}
H= & \sum_{i}\left(\varepsilon_{a} \hat{a}_{i}^{+} \hat{a}_{i}+\varepsilon_{b} \hat{b}_{i}^{+} \hat{b}_{i}+U \hat{n}_{a i} \hat{n}_{b i}\right)-\sum_{\langle i j\rangle}\left(t_{a} \hat{a}_{i}^{+} \hat{a}_{j}+t_{b} \hat{b}_{i}^{+} \hat{b}_{j}\right. \\
& + \text { H.c. }) .
\end{aligned}
$$

Here, the strong repulsion between ${ }^{4} \mathrm{He}$ atoms in close proximity is modeled by taking $\hat{a}$ and $\hat{b}$, field operators for ${ }^{4} \mathrm{He}$ atom in the $a$ and $b$ states, as hard-core boson operators. $U$ is the repulsion between a ${ }^{4} \mathrm{He}$ atom occupying $\phi_{a}$ and one occupying $\phi_{b}$ on the same site, and is of the order of the local interstitial activation energy. Of the various energies in the problem, this is by far the largest $(\sim 50 \mathrm{~K})$, and for the purpose of this work we will consider $U \rightarrow \infty$, so each site can at most be singly occupied by a ${ }^{4} \mathrm{He}$ atom. In other words, we neglect the possibility of interstitials. $t_{a}$ and $t_{b}$ denote the hopping amplitudes of the tunneling process between neighboring sites from $a$ to $a$ state and from $b$ to $b$ state, respectively ( $\langle i j\rangle$ indicates nearest neighbors). The $b$ boson has higher on-site energy $\left(\varepsilon_{b}>\varepsilon_{a}\right)$, but also a higher hopping amplitude $\left(t_{b}>t_{a}\right)$. The hard-core nature of the bosons implies that hopping is only possible between an occupied site and a neighboring vacancy.

In the torsional oscillator experiment setup used to observe NCRI, the density of ${ }^{4} \mathrm{He}$ atoms is held fixed. However, because the lattice constant of the solid is determined by minimizing the free energy, the number of bosons per site $\left\langle\hat{n}_{a}\right\rangle+\left\langle\hat{n}_{b}\right\rangle$ is not externally imposed. Within this model, if $\left\langle\hat{n}_{a}\right\rangle+\left\langle\hat{n}_{b}\right\rangle=1$, the ${ }^{4} \mathrm{He}$ atoms form a commensurate solid. If further $\left\langle\hat{n}_{b}\right\rangle=0$, the commensurate solid is DF, while if $\left\langle\hat{n}_{a}\right\rangle$ $=0$, it is an exciton solid. However, if $\left\langle\hat{n}_{a}\right\rangle+\left\langle\hat{n}_{b}\right\rangle<1$, then the solid is an incommensurate one with a finite vacancy density. The excitation energy of a single vacancy from the DF state is $\left|\varepsilon_{a}\right|-z t_{a}$, where $z$ is the coordination number. The Andreev-Lifshitz vacancy mechanism for supersolidity would require this to be negative, which evidently is not the case. On the other hand, the instability criterion for generating a vacancy above the exciton solid is $-\varepsilon_{b}-z t_{b}$, which is easier to satisfy. Vacancies can then Bose condense above the exciton background and the condensation energy gain may be sufficient to overcome the exciton energy $\Delta=\varepsilon_{b}-\varepsilon_{a}$. Should this be the case, the DF state will be metastable. In their theory, DMZ identified the DF state as the normal solid at $T=0$, and the exciton state with Bose condensation of vacancies as the supersolid. Using a single-site mean field theory, DMZ showed that as parameters in the mode are tuned (corresponding experimentally to, for example, changing the pressure), there is a transition at $T=0$ from the DF normal solid to the supersolid state described above. Since the DF state is metastable, this transition is naturally first order, and involves a jump not only in the Bose condensed amplitude but also in the densities of both vacancies and exciton defects. Hence the supersolid transition is accompanied by a commensurate-incommensurate transition, as well as a change in local density profile of the ${ }^{4} \mathrm{He}$ atoms. Because the normal solid is commensurate, it explains why experimental measurements performed on the normal solid do not show an appreciable density of vacancies.

Because the results of DMZ are based on a single-site mean field theory, it is of interest to examine if they hold up 
against the effects of both quantum and thermal fluctuations. At low temperature, the dominating effects should be due to phase fluctuations of the condensate, since they correspond to gapless modes. Experimentally, at the lowest temperature, NCRI in solid ${ }^{4} \mathrm{He}$ is observed even at the highest pressure achievable in the laboratory. Thus, the $T=0$ first-order supersolid-normal solid transition predicted by DMZ cannot be tested at present. It is therefore important to determine the nature of the supersolid-normal solid transition at finitetemperature within DMZ's theory to compare to experiments. We confirm that when phase fluctuations are included, the finite-temperature transition remains first order for system parameters not too far from the zero-temperature transition point. Moreover, we find that fluctuations give rise to a "reentrance" phenomenon.

\section{B. Trapped Bosons in Optical Lattice}

For solid ${ }^{4} \mathrm{He}$, the lattice constant is self-adjusted to minimize the free energy. We can also consider a system of bosons in the presence of an external periodic potential, in which case the lattice constant will be externally imposed. An interesting realization of such a system has been achieved recently in ultracold trapped bosons in an optical lattice., ${ }^{4,24}$ By superimposing counterpropagating laser beams of wavelength $\lambda$ in different directions, an effective periodic potential in one, two, or three dimensions can be produced. In three dimensions, the potential has the form

$$
V(x, y, z)=V_{0 x} \cos ^{2}(k x)+V_{0 y} \cos ^{2}(k y)+V_{0 z} \cos ^{2}(k z),
$$

where $k=2 \pi / \lambda$ is the wave number, and the strengths of the potential in the three directions are proportional to the laser intensity in each direction and can therefore be tuned. For a single particle, this periodic potential gives rise to the usual energy bands in the first Brillouin zone. For $V_{0}$ large compared to the so-called recoil energy $E_{R}=\hbar^{2} k^{2} / 2 m$, the lower bands can be viewed as tight-binding bands arising from a periodic array of harmonic wells. For deep potential of cubic symmetry $\left(V_{0 x}=V_{0 y}=V_{0 z}=V_{0}\right)$, the Wannier basis for these bands can to a good approximation be constructed from the eigenstates of the spherically symmetric harmonic oscillator potential $V_{h a r}(r)=V_{0} k^{2} r^{2}$. The bands can then be denoted by the quantum numbers $\left(n_{x}, n_{y}, n_{z}\right)$ of these eigenstates, and their eigenenergies $\left(n_{x}+n_{y}+n_{z}+1 / 2\right) \hbar \omega_{0}$, where $\hbar \omega_{0}$ $=\sqrt{4 V_{0} E_{R}}$ provide approximate values for the band gaps. It is convenient to consider the optical lattice experiments as being performed at fixed chemical potential. In the case where $\hbar \omega_{0}$ is much larger than other relevant energy scales, all the particles will occupy the lowest band, and the system can be modeled by a single-band boson Hubbard model: ${ }^{25}$

$$
\begin{aligned}
H= & \sum_{i} \varepsilon_{a} \hat{a}_{i}^{+} \hat{a}_{i}+U \sum_{i} \hat{n}_{a i}\left(\hat{n}_{a i}-1\right)-\sum_{\langle i j\rangle}\left(t_{a} \hat{a}_{i}^{+} \hat{a}_{j}+\text { H.c. }\right) \\
& -\mu \sum_{i}\left(\hat{a}_{i}^{+} \hat{a}_{i}\right)
\end{aligned}
$$

with $\mu$ as the chemical potential. This is a well-studied model, and it has been established that with increasing $t_{a} / U$, there is a quantum phase transition from a Mott insulator state with integer filling per site to a SF state. The SF state may have commensurate or incommensurate filling depending on $\mu$. Trapped bosons in the optical lattice provide an elegant realization of this model which allows the transition to be studied systematically in experiments.

Alternatively, we can consider the limit where the Hubbard $U$ is the dominating energy. This can be achieved experimentally by tuning close to the Feshbach resonance. In this case the Mott-Hubbard gap prevents any double or higher occupancy $n_{i} \leqslant 1$, and the bosons are hard core. If we restrict to the lowest band still, then the ground state is an insulator for $\mu<\varepsilon_{a}-z t_{a}$ (vacuum) and $\mu>\varepsilon_{a}+z t_{a}$ (one boson on each site), while if $\mu$ lies between these two limits, the system is a superfluid with $\left\langle n_{i}\right\rangle$ changing continuously from 0 to 1 as $\mu$ increases. Unlike the MI-SF transition with changing $t_{a} / U$, these insulator-SF transitions caused by changing $\mu$ in the hard-core model are just by-products of density transitions.

For $\mu>\varepsilon_{a}+z t_{a}$, one has essentially a filled band insulator if we restrict to the lowest band. However, if the higher bands are considered, then it is possible for bosons to delocalize and form a SF. We emphasize from the start that this is not simply due to partially occupying the higher bands while keeping the lowest band filled or even transferring some bosons from the lowest band to a higher band, as the hardcore condition would still forbid any boson motion if every site is singly occupied independent of which band it is in. Instead the boson delocalization can only occur by introducing vacancies.

We consider the case where in addition to the lowest band, we include one higher band, with both strong enough intraband and interband on-site repulsion between bosons to make them hard core. One thus arrives at a two-band boson model

$$
\begin{aligned}
H= & \sum_{i}\left(\varepsilon_{a} \hat{a}_{i}^{+} \hat{a}_{i}+\varepsilon_{b} \hat{b}_{i}^{+} \hat{b}_{i}\right)-\sum_{\langle i j\rangle}\left(t_{a} \hat{a}_{i}^{+} \hat{a}_{j}+t_{b} \hat{b}_{i}^{+} \hat{b}_{j}+\text { H.c. }\right) \\
& -\mu \sum_{i}\left(\hat{a}_{i}^{+} \hat{a}_{i}+\hat{b}_{i}^{+} \hat{b}_{i}\right),
\end{aligned}
$$

with the constraint $n_{a i}+n_{b i} \leqslant 1$. This Hamiltonian is of the same form as the DMZ two-band model for supersolid except for the presence of the chemical potential term, which can simply be absorbed into a redefinition of $\varepsilon_{a}$ and $\varepsilon_{b}$. The $\hat{a}$ and $\hat{b}$ operators are for the lowest and the higher bands, respectively. In analogy to the supersolid model, we will refer to a boson in the $b$ band as an exciton. Because the Wannier state for the higher band is less localized, the hopping amplitude $t_{b}$ will have a bigger magnitude than $t_{a}$ if the lattice constant $\lambda / 2$ is sufficiently large. The ratio of the hopping amplitudes can be estimated by looking at the ratio of overlaps between states on neighboring sites. Using this estimate, we calculate the quantity $t_{m n} / t_{00}$, where $t_{m n}$ is the tunneling amplitude from eigenstate $n$ of a well into eigenstate $m$ of a neighboring well. In Fig. 1(a), we plot $t_{00}$ as a function of the distance between neighboring harmonic oscillator wells. The dependence of the ratio $t_{m m} / t_{00}$ on the distance is shown in Fig. 1(b) for $m=0,1,2$ in one dimension. If we identify $a$ as the $n=0$ state, and $b$ as either the 

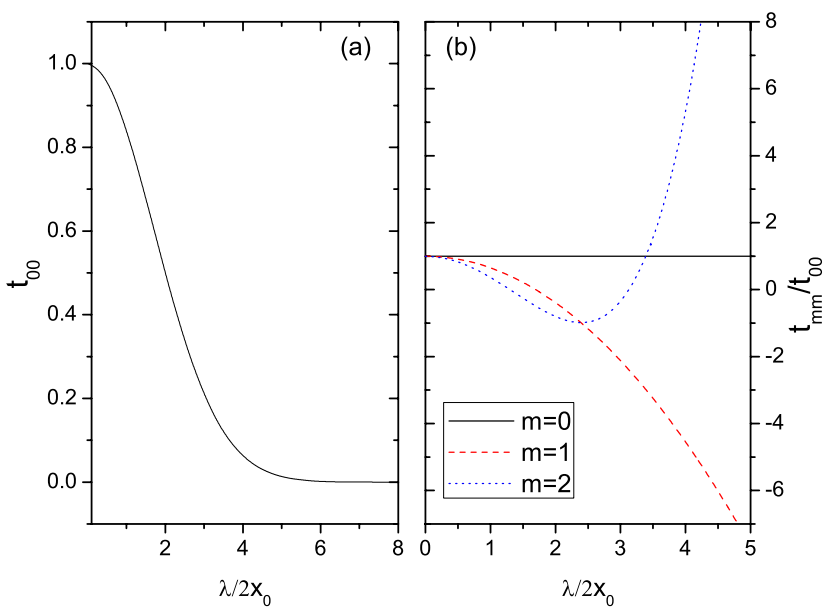

FIG. 1. (Color online) (a) The overlap $t_{00}$ and (b) $t_{m m}(m$ $=0,1,2)$ scaled by $t_{00}$, as functions of the distance $\lambda / 2$ between two neighboring centers in unit of the Gaussian half-width $x_{0}$. We use $t_{m n}(a)=\int_{-\infty}^{\infty} \varphi_{m}^{*}(x) \varphi_{n}(x-a) d x$, where $\varphi_{n}(x-a)$ denotes the eigenstate of the $n$th energy level of the harmonic oscillator with center at $x$ $=a$.

$n=1$ or $n=2$ state, then we can see clearly that $t_{b}$ can become significantly larger than $t_{a}$ as $\lambda / 2 x_{0}$ increases, where $x_{0}$ is the Gaussian half-width of the $n=0$ harmonic oscillator eigenstate.

Instead of the lowest and a higher band of a single type of bosons, the two-band model also acts as the model for the case of two different types of bosons $(a, b)$ each restricted to its respective lowest band in the optical lattice. For example, we may have bosons of two different masses $\left(m_{a}>m_{b}\right)$. If we assume the laser beams produce the same periodic potential on them, then we have $\Delta=\varepsilon_{b}-\varepsilon_{a}=\hbar \omega_{0 b}-\hbar \omega_{0 a}$, where $\hbar \omega_{0 a, b} \propto \sqrt{\frac{1}{m_{a, b}}}$ are the harmonic oscillator frequencies for masses $m_{a}$ and $m_{b}$, respectively. We also have $t_{b}>t_{a}$, due to larger zero point motion of the $b$ boson. However, because the tunneling amplitude $t_{00}^{a, b}$ is exponentially sensitive to the Gaussian half-width of the harmonic oscillator wave function, we expect the difference in tunneling amplitudes to be the more significant effect.

Before we discuss our calculation for the two-band model, we should clarify the terminology used below. In both solid ${ }^{4} \mathrm{He}$ and trapped bosons in optical lattice, Galilean invariance is violated. In the optical lattice case, the lack of translational invariance is externally imposed, so when bosons Bose condense, we consider it to be a superfluid, not a supersolid. In the case of solid ${ }^{4} \mathrm{He}$, the translational invariance is spontaneously broken by the solid, and if Bose condensation occurs without the solid melting, we have a supersolid, to be distinguished from the superfluid, which refers to the situation in the liquid. However, since we are not interested in the melting transition, and since the two-band model is applied to both solid ${ }^{4} \mathrm{He}$ and optical lattice, we will simply refer to the Bose condensed phase as the SF phase in both systems for convenience in what follows.

\section{MODIFIED MEAN FIELD THEORY}

Our primary goal is to analyze the above two-band boson Hubbard model to include phase fluctuations. Since we do not expect the precise details of the individual parameters in the Hamiltonian other than the gross features described above to be crucial, we take $t_{a}=0$ for convenience. The boson model can then be mapped onto a spin $S=1 / 2 X Y$ model coupled to an Ising degree of freedom as follows:

$$
\begin{aligned}
H= & \sum_{i}\left(\varepsilon_{a}-\mu\right)+\sum_{i}\left(\Delta+\frac{h}{2}\right) \hat{n}_{i}-h \sum_{i} \hat{S}_{i z} \hat{n}_{i} \\
& -t_{b} \sum_{\langle i j\rangle}\left(\hat{S}_{i}^{+} \hat{S}_{j}^{-}+\hat{S}_{i}^{-} \hat{S}_{j}^{+}\right) \hat{n}_{i} \hat{n}_{j},
\end{aligned}
$$

with $h=\mu-\left(\varepsilon_{a}+\Delta\right)$. The mapping between the original boson operators and the new set of operators $\hat{n}$ and $\hat{S}$ is

$$
\begin{gathered}
\hat{n}_{i}|0\rangle=|0\rangle, \quad \hat{n}_{i} \hat{b}_{i}^{+}|0\rangle=\hat{b}_{i}^{+}|0\rangle, \quad \hat{n}_{i} \hat{a}_{i}^{+}|0\rangle=0, \\
\hat{S}_{i}^{z}=\hat{b}_{i}^{+} \hat{b}_{i}-\frac{1}{2}, \quad \hat{S}_{i}^{+}=\hat{b}_{i}^{+}, \quad \hat{S}_{i}^{-}=\hat{b}_{i},
\end{gathered}
$$

where $|0\rangle$ is the empty site, i.e., vacancy state. The Ising operator $\hat{n}_{i}$ has eigenvalues 0 and 1 . For bosons, it acts as the defect (vacancy or exciton) occupation number operator. The spin-1/2 operator $\hat{S}_{i}$ acts on the Hilbert space of the $|0\rangle$ (vacancy) and $\hat{b}_{i}^{+}|0\rangle$ (exciton) doublet, which is assumed to be closer to each other in energy than to that of $\hat{a}_{i}^{+}|0\rangle$.

Because we take $t_{a}=0$, the $a$ bosons cannot Bose condense, and the Bose condensation order parameter, taken to be real, is given by $\left\langle\hat{b}_{i}\right\rangle=\left\langle\hat{b}_{i}^{+}\right\rangle=\left\langle\hat{S}_{i x}\right\rangle$. In this representation, the single-site mean field theory (MFT) of DMZ would correspond to approximating $\hat{S}_{i}^{+} \hat{S}_{j}^{-} \hat{n}_{i} \hat{n}_{j}$ by

$$
\begin{aligned}
\hat{S}_{i}^{+} \hat{S}_{j}^{-} \hat{n}_{i} \hat{n}_{j} \approx & \hat{S}_{i}^{+}\left\langle\hat{S}_{j}^{-}\right\rangle\left\langle\hat{n}_{i}\right\rangle\left\langle\hat{n}_{j}\right\rangle+\hat{S}_{j}^{-}\left\langle\hat{S}_{i}^{+}\right\rangle\left\langle\hat{n}_{i}\right\rangle\left\langle\hat{n}_{j}\right\rangle+\hat{n}_{i}\left\langle\hat{S}_{i}^{+}\right\rangle\left\langle\hat{S}_{j}^{-}\right\rangle\left\langle\hat{n}_{j}\right\rangle \\
& +\hat{n}_{j}\left\langle\hat{S}_{i}^{+}\right\rangle\left\langle\hat{S}_{j}^{-}\right\rangle\left\langle\hat{n}_{i}\right\rangle-3\left\langle\hat{S}_{i}^{+}\right\rangle\left\langle\hat{S}_{j}^{-}\right\rangle\left\langle\hat{n}_{i}\right\rangle\left\langle\hat{n}_{j}\right\rangle .
\end{aligned}
$$

If we apply the single-site mean field theory to this model, we simply recover the results of DMZ for the choice of parameters used here. Such MFT of course neglects fluctuations completely, and it is legitimate to question if the results remain valid when fluctuations are included. At low temperature, the dominating fluctuations in three dimensions should be those from gapless excitations, which are the phase modes of the Bose condensate, or spin waves in the spin language. In order to include the effects of phase fluctuations, we modify the mean field theory as follows:

$$
\begin{aligned}
\hat{S}_{i} \hat{S}_{j} \hat{n}_{i} \hat{n}_{j}= & \left\langle\hat{S}_{i} \hat{S}_{j}\right\rangle\left(\left\langle\hat{n}_{i}\right\rangle \hat{n}_{j}+\hat{n}_{i}\left\langle\hat{n}_{j}\right\rangle\right)+\hat{S}_{i} \hat{S}_{j}\left\langle\hat{n}_{i}\right\rangle\left\langle\hat{n}_{j}\right\rangle \\
& -2\left\langle\hat{S}_{i} \hat{S}_{j}\right\rangle\left\langle\hat{n}_{i}\right\rangle\left\langle\hat{n}_{j}\right\rangle .
\end{aligned}
$$

That is, correlations between the Ising degrees of freedom on different sites and between the Ising and spin degrees of freedom on the same or different sites are still ignored. However, correlations between the spin degrees of freedom on different sites will be included to allow for spin-wave physics. The modified mean field Hamiltonian can now be written as

$$
H=H_{n}+H_{s}+C
$$

where 


$$
\begin{aligned}
& H_{s}=-h \bar{n} \sum_{i} \hat{S}_{i}^{z}-J \sum_{\langle i j\rangle}\left(\hat{S}_{i}^{x} \hat{S}_{j}^{x}+\hat{S}_{i}^{y} \hat{S}_{j}^{y}\right), \\
& H_{n}=\left(\Delta+\frac{h}{2}-h M-2 t_{b} Z \bar{n} B\right) \sum_{i} \hat{n}_{i}, \\
& C=\sum_{i}\left[\left(\varepsilon_{a}-\mu\right)+h \bar{n} M+2 t_{b} Z \bar{n}^{2} B\right],
\end{aligned}
$$

where $J=2 t_{b} \bar{n}^{2}$ and $Z$ is the coordination number.

$$
\begin{gathered}
M=\left\langle\hat{S}_{i}^{z}\right\rangle, \\
B=\left\langle\hat{S}_{i}^{x} \hat{S}_{i+\delta}^{x}\right\rangle+\left\langle\hat{S}_{i}^{y} \hat{S}_{i+\delta}^{y}\right\rangle, \\
\bar{n}=\left\langle\hat{n}_{i}\right\rangle
\end{gathered}
$$

are parameters independent of the site number and to be determined self-consistently. It can be seen that the spin part Hamiltonian $H_{s}$ is a ferromagnetic $X Y$ model in a transverse field with exchange coupling $J$.

$H_{n}$ is a single-site Hamiltonian and $\left\langle\hat{n}_{i}\right\rangle$ is easily calculated. The self-consistent equation for $\bar{n}$ is then given by

$$
\bar{n}=\frac{\operatorname{Tr}\left[n_{i} e^{-\beta H}\right]}{\operatorname{Tr}\left[e^{-\beta H}\right]}=\frac{\exp \left(-\beta E_{1}\right)}{\frac{1}{2}+\exp \left(-\beta E_{1}\right)},
$$

where

$$
E_{1}=\Delta+\frac{h}{2}-h M-2 t_{b} Z \bar{n} B
$$

The factor $\frac{1}{2}$ in the denominator arises from the $n_{i}=0$ state being a singlet state while the $n_{i}=1$ state is a doublet.

The most important feature of $H_{s}$ is its continuous $X Y$ symmetry which gives rise to gapless excitations (Goldstone modes) in the ordered state. This physics will not be affected by the transverse field $h$ provided $|h|$ is not too big. Thus, as further simplification, we consider in this work the zero field case, or in other words the case where the $b$ state and the vacancy are degenerate. Setting $h=0$, the three parts of the MF Hamiltonian become

$$
\begin{gathered}
H_{s}=-J \sum_{\langle i j\rangle}\left(\hat{S}_{i}^{x} \hat{S}_{j}^{x}+\hat{S}_{i}^{y} \hat{S}_{j}^{y}\right), \\
H_{n}=\left(\Delta-2 t_{b} Z \bar{n} B\right) \sum_{i} \hat{n}_{i}, \\
C=N\left[\left(\varepsilon_{a}-\mu\right)+2 t_{b} Z \bar{n}^{2} B\right] .
\end{gathered}
$$

\section{Modified spin-wave method}

After the modified mean field decoupling, $H_{s}$ remains a many body Hamiltonian. Since our main goal is to include the effects of the condensate phase fluctuations, which in the spin language implies fluctuations of spin waves, it is natural to use the spin-wave approximation. The spin-wave theory is known to be reliable in three dimensions, where long range order persists at low temperature. However, it is seen that the coupling $J$ is proportional to $\bar{n}^{2}$, which is itself temperature dependent, and as we will see, will imply that we need to address $H_{s}$ both below and above the spin ordering temperature. One possible method that can be used is the Schwinger boson MFT, but it has been shown that this method is problematic for temperature comparable or larger than $J .^{26}$ Instead, we use a modified spin wave method similar to the approaches introduced by Takahashi ${ }^{28,29}$ and by Hirsch and Tang. 30

The calculation will be performed on the threedimensional (3D) cubic lattice. For the case of bosons on the optical lattice, this is the actual lattice that has been studied experimentally. For solid ${ }^{4} \mathrm{He}$, the lattice is hcp, but since $H_{s}$ is ferromagnetic, the physics does not depend on the precise lattice structure beyond the coordination number $Z$. To perform the spin-wave theory, it is convenient ${ }^{27}$ to globally rotate the spins about the spin $y$ axis and rewrite $H_{s}$ as

$$
H_{s}=-J \sum_{l \delta}\left(\hat{P}_{l}^{z} \hat{P}_{l+\delta}^{z}+\hat{P}_{l}^{x} \hat{P}_{l+\delta}^{x}\right)
$$

where the $\hat{P}$ 's are spin operators in the rotated frame. The classical ground state can then be taken as having all the spins pointing in the $+z$ direction. Next, the HolsteinPrimakoff transformation is defined

$$
\begin{gathered}
P_{l}^{+}=\sqrt{2 S-c_{l}^{+} c_{l}} c_{l}, \\
P_{l}^{-}=c_{l}^{+} \sqrt{2 S-c_{l}^{+} c_{l}}, \\
P_{l}^{z}=S-c_{l}^{+} c_{l} .
\end{gathered}
$$

The linearized spin-wave Hamiltonian $H_{S W}$ is obtained by expanding the square roots and keeping to quadratic order in the bosonic $c$ operators. This quadratic Hamiltonian can then be solved using Bogliubov transformation

$$
d_{k}=\cosh \theta_{k} c_{k}-\sinh \theta_{k} c_{-k}^{+}, \quad d_{k}^{+}=\cosh \theta_{k} c_{k}^{+}-\sinh \theta_{k} c_{-k},
$$

where

$$
c_{l}=\frac{1}{\sqrt{N}} \sum_{k} e^{i k \cdot l} c_{k}, \quad c_{l}^{+}=\frac{1}{\sqrt{N}} \sum_{k} e^{-i k \cdot l} c_{k}^{+} .
$$

Unlike the Heisenberg ferromagnet, where the classical ground state is also the quantum mechanical one, quantum fluctuations are present for the $X Y$ ferromagnet. As a result $\left\langle P_{l}^{z}\right\rangle<1 / 2$ even for the ground state. Provided the difference is small though, the classical ground state can be considered to be a good approximation and the spin-wave theory should be reliable. As temperature increases, however, the amount of fluctuations increases, and eventually $\left\langle P_{l}^{z}\right\rangle$ calculated by spin-wave theory becomes $<0$, which clearly indicates spinwave theory ceases to be valid. However, spin-wave theory can be applied if a Lagrangian multiplier or magnon chemical potential $\lambda$ is added to restrict the total number of magnons excited. The modified spin-wave Hamiltonian is given by 


$$
H_{S W}^{\prime}=H_{S W}-\lambda \sum_{l}\left(S-c_{l}^{+} c_{l}\right)
$$

with the provision that if $\left\langle c_{l}^{+} c_{l}\right\rangle \leqslant 1 / 2$ when calculated without $\lambda$, then $\lambda=0$, and if $\left\langle c_{l}^{+} c_{l}\right\rangle>1 / 2$, then $\lambda$ becomes nonzero and is adjusted to enforce $\left\langle c_{l}^{+} c_{l}\right\rangle=1 / 2$, i.e., $\left\langle P_{l}^{z}\right\rangle=0$. After Bogliubov transformation, $H_{S W}^{\prime}$ becomes diagonalized

$$
H_{S W}^{\prime}=-N Z \frac{3 J}{8}-\lambda N+\sum_{k} E_{k}\left(d_{k}^{+} d_{k}+\frac{1}{2}\right),
$$

where the magnon excitation energy

$$
E_{k}=\frac{J}{2} Z\left(1+\lambda^{\prime}\right) \sqrt{1-\frac{\gamma_{k}}{\left(1+\lambda^{\prime}\right)}}
$$

and $\lambda^{\prime}=\frac{2 \lambda}{J}, \quad \gamma_{k}=\frac{1}{Z} \sum e^{i k \cdot \delta}$. The Bogliubov coefficients are found to be

$$
\cosh 2 \theta_{k}=\frac{\alpha_{k}}{E_{k}}, \quad \sinh 2 \theta_{k}=\frac{2 \beta_{k}}{E_{k}},
$$

with

$$
\alpha_{k}=t_{b} Z \bar{n}^{2}-\frac{1}{2} t_{b} Z \bar{n}^{2} \gamma_{k}+\lambda, \quad \beta_{k}=\frac{1}{4} t_{b} Z \bar{n}^{2} \gamma_{k} .
$$

The Bose condensed order parameter is

$$
\langle\hat{b}\rangle=\left\langle P^{z}\right\rangle=1-\frac{1}{N} \sum_{k} \cosh 2 \theta_{k}\left(n_{k}+\frac{1}{2}\right),
$$

where

$$
n_{k}=\left\langle d_{k}^{+} d_{k}\right\rangle=\left[\exp \left(\beta E_{k}\right)-1\right]^{-1}
$$

is the Bose-Einstein distribution of the magnons, $\beta^{-1}=T$ (we pick energy unit so that the Boltzmann constant $k_{B}=1$ ). The dependence of $\langle\hat{b}\rangle$ on $\bar{n}$ as calculated from normal (linearized) spin-wave theory with and without the Lagrange multiplier at a fixed temperature is shown in Fig. 2(a). When the order parameter $\langle\hat{b}\rangle$ is positive, denoting the SF phase, $\lambda$ $=0$ and the dispersion $E_{k}$ represents a gapless mode and is linear in $k$ at small wave number $k$. On the contrary, the excitation energy $E_{k}$ has a finite gap for nonzero $\lambda$ and hence for zero $\langle\hat{b}\rangle$, indicating a MI phase.

Before we set off to calculate the self-consistent parameters $B$ and $\bar{n}$, a glance at the bosonic distribution $n_{k}$ alerts us that the spin-wave method with chemical potential is still problematic. Since $J \propto \bar{n}^{2}$, the effective spin temperature is $T^{\prime}=T / \bar{n}^{2}$. Though the Lagrangian multiplier prescription is reliable up to $T \sim J=2 t_{b} \bar{n}^{2}$, it fails when $T \gg J$. However, the necessity to consider small $\bar{n}$ values can mean $T \gg J$ even at low temperature. One major problem with the modified spinwave method discussed so far is that at high $T^{\prime}$, the nearest neighbor spin-spin correlation function $B$ can actually take on the wrong sign. This problem has been recognized in literature, and a remedy has been proposed. ${ }^{28,29}$ The correct sign of $B$ can be maintained if when calculating the spin correlation $\left\langle P_{l}^{z} P_{l+\delta}^{z}+P_{l}^{x} P_{l+\delta}^{x}\right\rangle$, one keeps not just the quadratic, but the quartic order in Holstein-Primakoff boson operators:
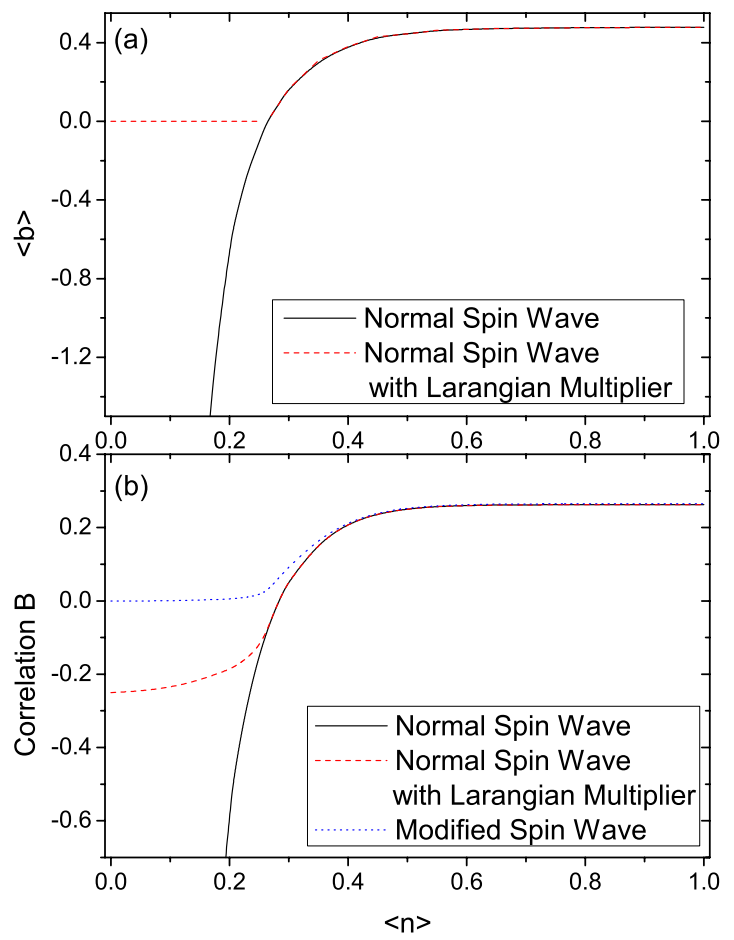

FIG. 2. (Color online) Comparisons between different spinwave approximations used to calculate the spin Hamiltonian [Eq. (10)] with coupling $J=2 t_{b} \bar{n}^{2}$. (a) The superfluid order parameter $\langle b\rangle$ and (b) the spin correlation $B=\left\langle S_{i}^{x} S_{j}^{x}+S_{i}^{y} S_{j}^{y}\right\rangle$ as functions of $\bar{n}$ at $k T=0.05 t_{b} Z$. $\langle b\rangle$ is tuned to be non-negative at small $\vec{n}$ 's by the Lagrangian multiplier. The correlation $B$ is further corrected by the modified spin-wave method.

$$
\begin{aligned}
\left\langle P_{l}^{z} P_{l+\delta}^{z}+P_{l}^{x} P_{l+\delta}^{x}\right\rangle= & P^{2}-P\left[\left\langle c_{i}^{+} c_{i}\right\rangle+\left\langle c_{j}^{+} c_{j}\right\rangle-\frac{1}{2}\left(\left\langle c_{i} c_{j}\right\rangle+\left\langle c_{i}^{+} c_{j}^{+}\right\rangle\right.\right. \\
& \left.\left.+\left\langle c_{i} c_{j}^{+}\right\rangle+\left\langle c_{i}^{+} c_{j}\right\rangle\right)\right]+\left\langle c_{i}^{+} c_{i} c_{j}^{+} c_{j}\right\rangle \\
& -\frac{1}{8}\left[\left\langle c_{i}^{+} c_{i} c_{i} c_{j}\right\rangle+\left\langle c_{i} c_{j}^{+} c_{j} c_{j}\right\rangle+\left\langle c_{i}^{+} c_{i}^{+} c_{i} c_{j}^{+}\right\rangle\right. \\
& +\left\langle c_{i}^{+} c_{j}^{+} c_{j}^{+} c_{j}\right\rangle+\left\langle c_{i}^{+} c_{i} c_{i} c_{j}^{+}\right\rangle+\left\langle c_{i} c_{j}^{+} c_{j}^{+} c_{j}\right\rangle \\
& \left.+\left\langle c_{i}^{+} c_{i}^{+} c_{i} c_{j}\right\rangle+\left\langle c_{i}^{+} c_{j}^{+} c_{j} c_{j}\right\rangle\right]+O\left(P^{-1}\right) .
\end{aligned}
$$

The quartic terms of $c$ operators are then evaluated by Hartree-Fock (HF) decoupling

$$
\left\langle c_{i}^{+} c_{i} c_{j}^{+} c_{j}\right\rangle \approx\left\langle c_{i}^{+} c_{i}\right\rangle\left\langle c_{j}^{+} c_{j}\right\rangle+\left\langle c_{i}^{+} c_{j}\right\rangle\left\langle c_{i} c_{j}^{+}\right\rangle+\left\langle c_{i}^{+} c_{j}^{+}\right\rangle\left\langle c_{i} c_{j}\right\rangle .
$$

In the theory by Takahashi, these HF terms need to be determined self-consistently, in essence renormalizing the quadratic Hamiltonian $H_{S W}^{\prime}$. That is too complicated to do in our problem, so we just evaluate them using the unrenormalized $H_{S W}^{\prime}$, in line with the scheme proposed by Hirsch and Tang. ${ }^{30}$ Using $H_{S W}^{\prime}$, the HF terms can be derived as

$$
\begin{aligned}
& \left\langle c_{i}^{+} c_{j}\right\rangle=f\left(r_{i}-r_{j}\right)-\frac{1}{2} \delta_{i j}, \\
& \left\langle c_{i} c_{j}^{+}\right\rangle=f\left(r_{i}-r_{j}\right)+\frac{1}{2} \delta_{i j},
\end{aligned}
$$




$$
\left\langle c_{i} c_{j}\right\rangle=\left\langle c_{i}^{+} c_{j}^{+}\right\rangle=g\left(r_{i}-r_{j}\right),
$$

where

$$
\begin{aligned}
& f\left(r_{i}-r_{j}\right)=\frac{1}{N} \sum_{k} \exp \left[i k \cdot\left(r_{i}-r_{j}\right)\right] \cosh 2 \theta_{k}\left(\left\langle d_{k}^{+} d_{k}\right\rangle+\frac{1}{2}\right), \\
& g\left(r_{i}-r_{j}\right)=\frac{1}{N} \sum_{k} \exp \left[i k \cdot\left(r_{i}-r_{j}\right)\right] \sinh 2 \theta_{k}\left(\left\langle d_{k}^{+} d_{k}\right\rangle+\frac{1}{2}\right) .
\end{aligned}
$$

Consequently, the spin correlation becomes

$$
\begin{aligned}
B(\bar{n}, T)= & \left\langle P_{l}^{z} P_{l+\delta}^{z}+P_{l}^{x} P_{l+\delta}^{x}\right\rangle=\left\{S-\left[f(0)-\frac{1}{2}-\frac{1}{2} g(\delta)\right.\right. \\
& \left.\left.-\frac{1}{2} f(\delta)\right]\right\}^{2}+\frac{1}{4}[g(\delta)-f(\delta)]^{2}+\frac{1}{2}\left[g(\delta)-\frac{1}{2} g(0)\right]^{2} \\
& +\frac{1}{2}\left[f(\delta)-\frac{1}{2} g(0)\right]^{2}-\frac{1}{4} g(0)^{2} .
\end{aligned}
$$

Though $B$ does not appear in the form of a complete square as the case of Heisenberg ferromagnet, ${ }^{28}$ the inclusion of the quartic terms nevertheless ensures that it has the right sign at all effective spin temperature $T^{\prime}$ [see Fig. 2(b)]. Together with the equation for $\bar{n}$ Eq. (9), this equation for $B$ provides the self-equations for $\bar{n}$ and $B$.

Finally, within our MFT, the free energy per site is

$$
\begin{aligned}
G(T) & =-\frac{k T}{N} \ln \operatorname{Tr} \exp \left[-\beta\left(H_{n}+C+H_{s}\right)\right] \\
& =-k T \ln \left(\frac{1}{2}+e^{-\beta E_{1}}\right)+t_{b} Z \bar{n}^{2} B(\bar{n}, T)-T S_{s},
\end{aligned}
$$

where $S_{s}$ is the spin entropy. The free energy is useful in case where there is more than one MF solution to determine which is the most stable solution. Within our MF approach this is derived in principle from $H_{S W}^{\prime}$, which gives another problem. At high temperature, the spin entropy for a spin$1 / 2$ system saturates to $\ln 2$. However, using $H_{S W}^{\prime}$, the spin entropy is $S_{s}=\Sigma_{k}\left[\left(1+n_{k}\right) \ln \left(1+n_{k}\right)-n_{k} \ln n_{k}\right]$ and can exceed this saturated value due to large fluctuations even when the Legrange multiplier limits the average value of $n_{k}$. Since the effective spin temperature $T^{\prime}$ can be enormous at small $\bar{n}$, this problem is relevant when we need to compare the free energy of the $\bar{n} \approx 0$ to the $\bar{n} \approx 1 \mathrm{MF}$ solutions at temperature of interest. Our remedy for this problem is to impose an upper limit $S_{S}=k_{B} \ln 2$ when the calculated value of $S_{s}$ exceeds that value.

\section{RESULTS AND DISCUSSIONS}

The results obtained for the $3 \mathrm{D}$ simple cubic lattice are as follows. The main result will be that (i) the $T=0$ first-order transition obtained by DMZ in their single-site MFT remains robust with phase fluctuations; (ii) close to the $T=0$ transition point, the finite-temperature transition will also be first order; ${ }^{31}$ and (iii) the stabilization of the ordered phase gives rise to reentrance with increasing temperature into the SF phase on the MI side of the $T=0$ transition. We first present our results for bosons in optical lattice in which case the relevant free energy density to be minimized is the free energy per site with a fixed chemical potential $\mu$. Within our model, the experimental change in the strength in the periodic potential corresponds to changing $\Delta / t_{b} Z$.

At zero temperature, there are no magnons. Using spinwave approximation, the superfluid order parameter and the spin correlation are, respectively, $\langle b\rangle \simeq 0.478$ and $B^{*}$ $=B(\bar{n}, T=0) \simeq 0.265$ for all nonzero $\bar{n}$ 's. The self-consistent equation (9) and its $T=0$ limit given by Eq. (26) indicate that when $\Delta / t_{b} Z<B^{*}, \bar{n}=1$ will be the self-consistent solution that minimizes the total energy of the system, but when $\Delta / t_{b} Z>B^{*}$, the ground state will have $\bar{n}=0$. However, the $\bar{n}=1$ self-consistent solution branch does not disappear and hence is metastable until $\Delta / t_{b} Z$ increases past $2 B^{*}$. Thus, there is a first-order phase transition from the MI state to the SF state with decreasing $\Delta / t_{b} Z$. Accompanying the MI-SF transition is a jump in boson density (or equivalently, vacancy density) and exciton density. We thus recover the "vacuum switching" first-order transition obtained by $\mathrm{DMZ}$ using the single-site MFT. Within single-site MFT, the transition from MI to SF occurs at $\Delta / t_{b} Z=0.25$, which is less than our critical value of $\Delta / t_{b} Z=B^{*}$. This is the consequence of the SF phase being stabilized against the MI phase due to quantum phase fluctuations,

On the SF side, as the temperature rises from zero, thermal fluctuations will decrease the SF order parameter $\langle b\rangle$ both directly and also indirectly through the decrease in $\bar{n}$. At the same time, the decrease in spin correlation $B$ can drive the system from the defect rich $(\bar{n}$ close to 1$)$ to the defect poor ( $\bar{n}$ close to 0$)$ phase. As $T$ continues to increase, we can expect one of the two scenarios. The condensate amplitude may vanish while remaining in the defect rich phase, followed subsequently by a transition from the defect rich into the defect poor phase. Should that be the case, we expect $\langle b\rangle$ to go to 0 continuously. The other possibility is that there is only one transition, which primarily is from defect rich to defect poor, and when that happens, it drives the Bose condensed amplitude to 0 . Our calculation shows that it is the second case that is realized provided $\Delta / t_{b} Z$ is not too far from $B^{*}$. This is shown in Fig. 3 for $\Delta / t_{b} Z=0.26$. The selfconsistent solutions of $\bar{n}$ as $T$ is increased are shown in Fig. 3(a). In between the $\bar{n}$ values of the solutions that evolve from the $\bar{n}=0$ and $\bar{n}=1$ solutions, there is a third selfconsistent solution. This solution is unstable, corresponding to a local maximum in the free energy [Fig 3(b)]. The low and high $\bar{n}$ solutions are local minima in the free energy. At $T=0$, the high $\bar{n}$ solution is the global minimum, but with increasing $T$, the small $\bar{n}$ solution becomes the free energy minimum at a critical temperature $T_{c}$. Beyond $T_{c}$, the large $\bar{n}$ solution becomes metastable, but it remains a local minimum until a higher temperature where it disappears by merging with the unstable middle branch solution. We show this for $\Delta / t_{b} Z$ just slightly less than $B^{*}$ in Fig. 4 . The sequence of behaviors described above is typical of first-order transition. In the present case, a jump in $\bar{n}$ occurs at $T_{c}$. Just below $T_{c}$, $\bar{n}$ is large enough that the coupling $J$ in $H_{s}$ is sufficient for 

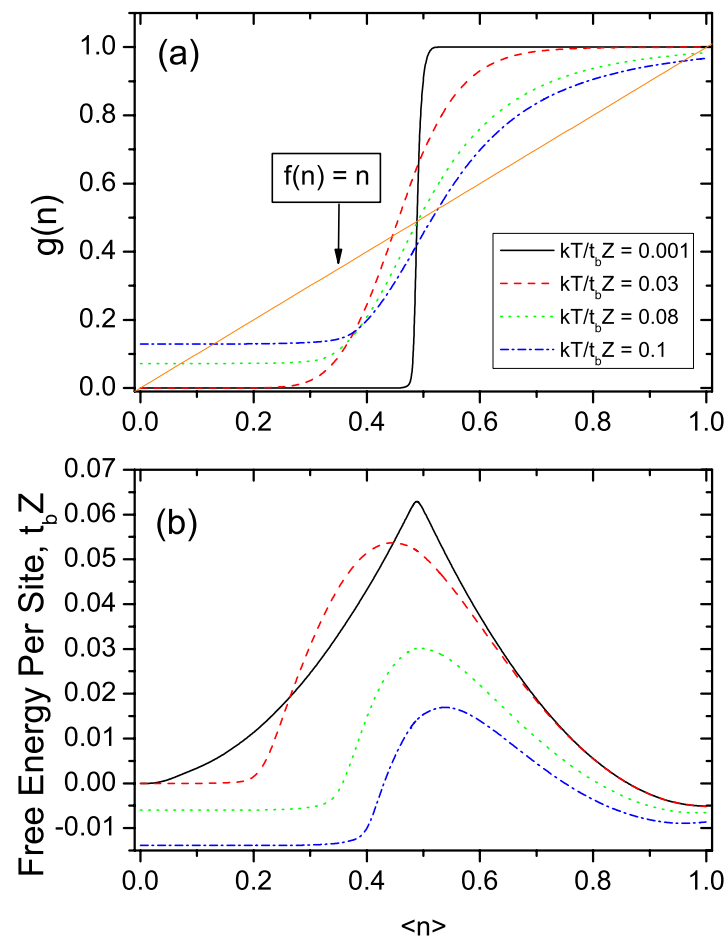

FIG. 3. (Color online) Mean field solutions of Eq. (9) at different temperatures for $\Delta / t_{b} Z=0.26$. (a) The self-consistent solutions occur where the curve $g(\bar{n})=\frac{\exp \left(-\beta E_{1}\right)}{\frac{1}{2}+\exp \left(-\beta E_{1}\right)}=\bar{n}$, where $\bar{n}$ is defect (vacancy and exciton) occupancy. The low and high $\bar{n}$ branches correspond, respectively, to the normal solid and supersolid solutions. (b) The free energy per site as a function of $\bar{n}$. The normal solid and supersolid solutions correspond to the two local minima. The firstorder SF-MI transition temperature is $T_{c} / t_{b} Z \simeq 0.083$.

$\langle b\rangle$ to be nonzero. Just above $T_{c}$, the jump to a small $\bar{n}$ results in a value of $J$ such that $T_{c}$ is above the Bose condensation temperature, and hence $\langle b\rangle$ jumps to 0 across the transition.

The value of $\Delta / t_{b} Z$ for Fig. 4 is very close to $B^{*}$, yet $T_{c}$ is not that close to 0 . The reason for this is because of the presence of reentrance in the MI side of the transition. The reentrance can be understood qualitatively as follows. Just on the MI side of the transition, the energy of the defect poor state and the defect rich state are almost degenerate. The defect poor phase has excitations with gap $\Delta$, and hence the difference of the free energy from the ground state energy is exponentially small at low $T$. The defect rich state, however, is a Bose condensate and has gapless excitations. Thus, its free energy decreases by a power of the temperature. As the temperature is raised, the free energy gain can overcome the ground state energy difference between the two phases, and the defect rich phase becomes more stable than the defect poor phase. Thus as the temperature is raised, the system first undergoes a (first-order) transition from the defect poor MI phase into the defect rich SF phase, and then later on at a higher temperature undergoes the SF-MI first-order transition discussed previously back into the defect poor phase. Since the reentrance is driven by gapless phase fluctuations, it will not occur if the $X Y$ spins are replaced by Ising spins, as in the so-called Blume-Capel model. ${ }^{32,33}$ For the same reason,
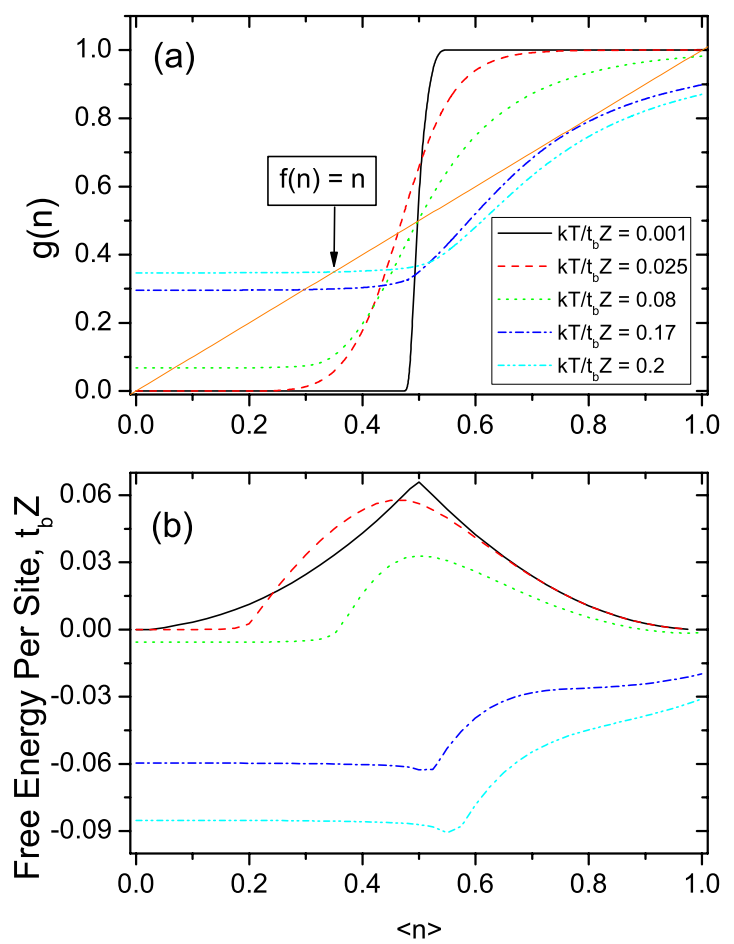

FIG. 4. (Color online) Same as Fig. 3, but for $\Delta / t_{b} Z=B^{*}-2$ $\times 10^{-7}$, where $B^{*}$ is the zero-temperature transition point. The firstorder SF-MI transition temperature is $T_{c} / t_{b} Z \simeq 0.0246$. The high $\bar{n}$ solution remains as a metastable minimum above $T_{c}$ and only disappears at a higher temperature.

approximate methods such as the single-site MFT that ignore phase fluctuations will necessarily miss the reentrance phenomenon.

On a more quantitative level, the free energy of the defect poor phase at low temperature is given by

$$
G_{0}(T)=-k_{B} T\left\{\ln \left[1+2 \exp \left(-\Delta / k_{B} T\right)\right]\right\} .
$$

On the other hand, for the defect rich phase, the dominant temperature correction is due to phase excitations about the condensate, giving

$$
G_{1}(T) \approx \Delta-t_{b} Z B^{*}-\frac{\zeta(3)}{\pi^{2}\left(t_{b} \bar{n}^{2} \sqrt{Z}\right)^{3}}\left(k_{B} T\right)^{4},
$$

where the Riemann zeta function $\zeta(3)=1.202$. If $G_{1}(T)$ $<G_{0}(T)$, then the stable phase is the defect rich Bose condensed phase. On the SF side $\left(\Delta<t_{b} Z B^{*}\right), G_{1}(0)<G_{0}(0)$, and the difference is further enhanced at low temperature due to thermal fluctuations. On the MI side, $G_{1}(0)>G_{0}(0)$, and the MI is the stable ground state. However, as $T$ increases, the $T^{4}$ correction in $G_{1}(T)$ dominates over the exponential correction in $G_{0}(T)$, and provided the ground state energy difference is not too big, $G_{1}(T)$ becomes smaller than $G_{0}(T)$ beyond some low temperature, giving rise to the "reentrance" phenomenon. As $T$ continues to increase, the disordering effect of thermal fluctuations finally dominates so that there is a second transition back into the defect poor MI phase. The temperature dependence of the free energies of 

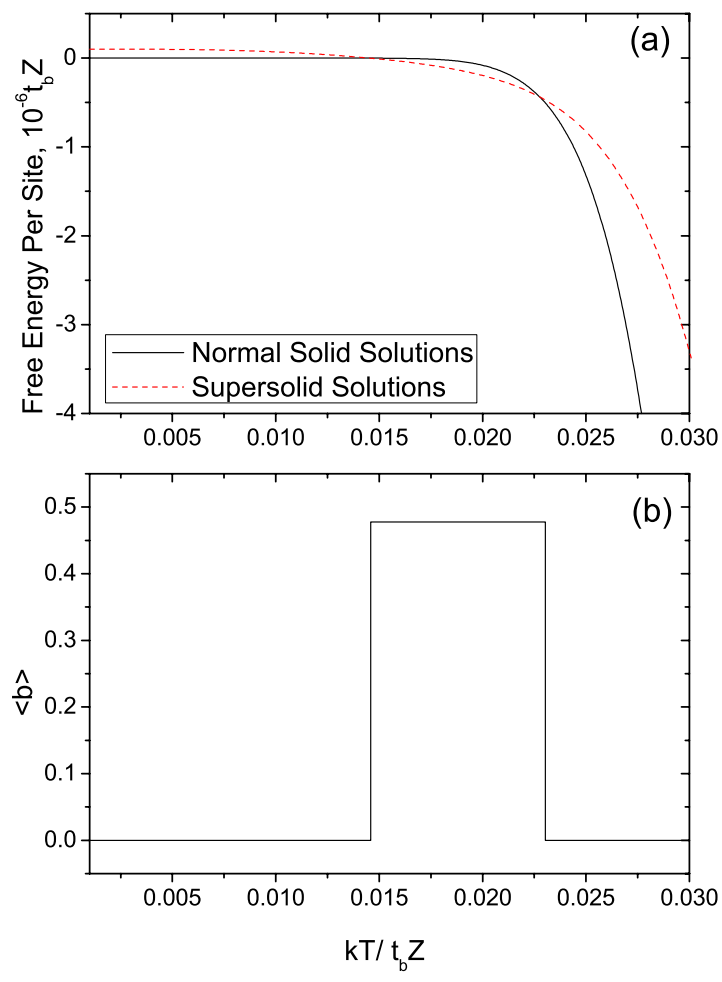

FIG. 5. (Color online) Figures showing reentrance for $\Delta / t_{b} Z$ $=B^{*}+10^{-7}$, where $B^{*}$ is the zero-temperature transition point. (a) The free energy per site plots of the normal solid and the supersolid self-consistent solutions as functions of temperature. There are two phase transitions, both first order, as the temperature increases for this specific $\Delta$. (b) The superfluid order parameter $\langle b\rangle$ as a function of temperature, showing sudden jumps at the two first-order phase transition points.

these two competing phases is illustrated in Fig. 5. The phase diagram in the vicinity of the $T=0$ SF-MI transition is shown in Fig. 6. The reentrance on the MI side can be clearly seen.

Although our results are based on our modified spin-wave theory for the spin Hamiltonian $H_{s}$, we believe the results are quite reliable. As a comparison, we consider the case of one dimensional, where $H_{s}$ can be solved exactly using the wellknown Jordan-Wigner transformation. The one-dimensional (1D) case by itself is also interesting because it can be realized experimentally in cold atoms. Using the Jordan-Wigner transformation, the spin-1/2 operators are mapped into spinless fermion operators

$$
\begin{gathered}
g_{l}=e^{i \phi_{l} S_{l}^{-},} \\
g_{l}^{+}=e^{-i \phi_{l} S_{l}^{+},} \\
g_{l}^{+} g_{l}=S_{l}^{+} S_{l}^{-}=\frac{1}{2}+S_{l}^{z},
\end{gathered}
$$

where

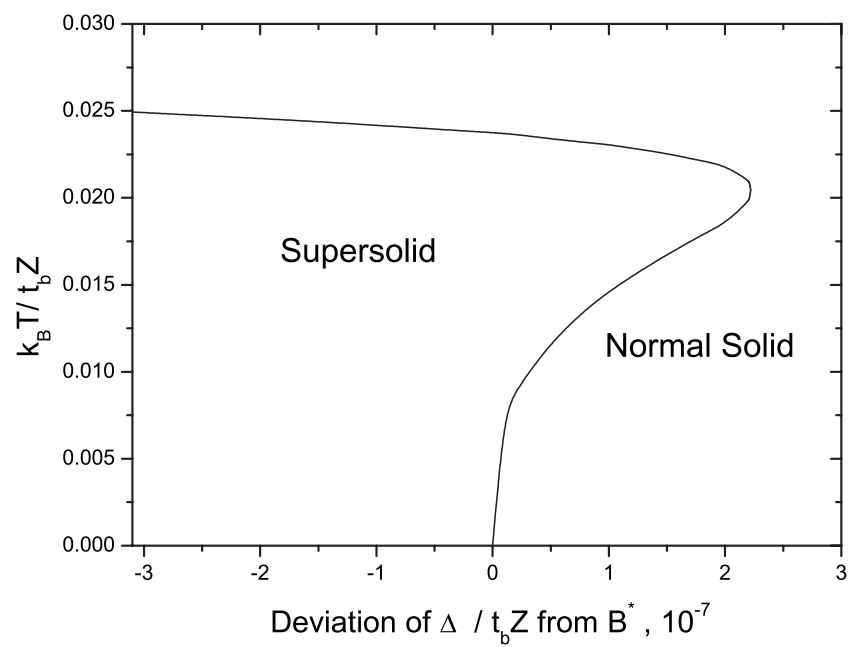

FIG. 6. The finite-temperature phase diagram close to the $T=0$ transition point $\Delta / t_{b} Z=B^{*} \simeq 0.265$, obtained by comparing free energy per site. There is a region on the zero-temperature normal solid side where the reentrance phenomenon occurs at finite temperature.

$$
\phi_{l}=\pi \sum_{i=1}^{l-1} g_{i}^{+} g_{i}
$$

and $\left\{g_{l}, g_{m}^{+}\right\}=\delta_{l m},\left\{g_{l}, g_{m}\right\}=0$. Then the spin Hamiltonian can be rewritten by the fermion $g$ operators and be diagonalized in $k$ space as

$$
H_{s}=-t_{b} \bar{n}^{2} \sum_{i}\left(\hat{S}_{i}^{+} \hat{S}_{i+1}^{-}+\hat{S}_{i}^{+} \hat{S}_{i-1}^{-}\right)=\sum_{k} \varepsilon_{k} g_{k}^{+} g_{k}
$$

with

$$
\varepsilon_{k}=-t_{b} Z \bar{n}^{2} \cos k
$$

The spin correlation is then found as

$$
B=\frac{1}{N} \sum_{k} \cos k\left\langle g_{k}^{+} g_{k}\right\rangle,
$$

where

$$
\left\langle g_{k}^{+} g_{k}\right\rangle=\left[\exp \left(\beta \varepsilon_{k}\right)+1\right]^{-1}
$$

is the fermion distribution function. At $T=0$, the negative energy levels are filled, while the positive ones are empty. The zero-temperature transition is at $\Delta / t_{b} Z=B^{* *}=\frac{1}{\pi} \approx 0.318$. We note that in addition to solving $H_{s}$ exactly, this result is actually the exact result for the Hamiltonian equation (2). This is because $\left[H, \hat{n}_{i}\right]=0$, so $n_{i}$ is a good quantum number for the ground state. Assuming no breaking of translational invariance, we then have either all $n_{i}=0$ or all $n_{i}=1$. Comparing the exact solution to the modified spin-wave method, which gives $\Delta / t_{b} Z=B_{1 D}^{*}=0.314$ as the transition point in one dimension, we see that the approximate modified spin-wave method does very well. We also note that both methods show that there is no Bose condensation in one dimension at $T$ $=0$, and the transition in $\bar{n}$ is driven by short range spin correlation $B$. 

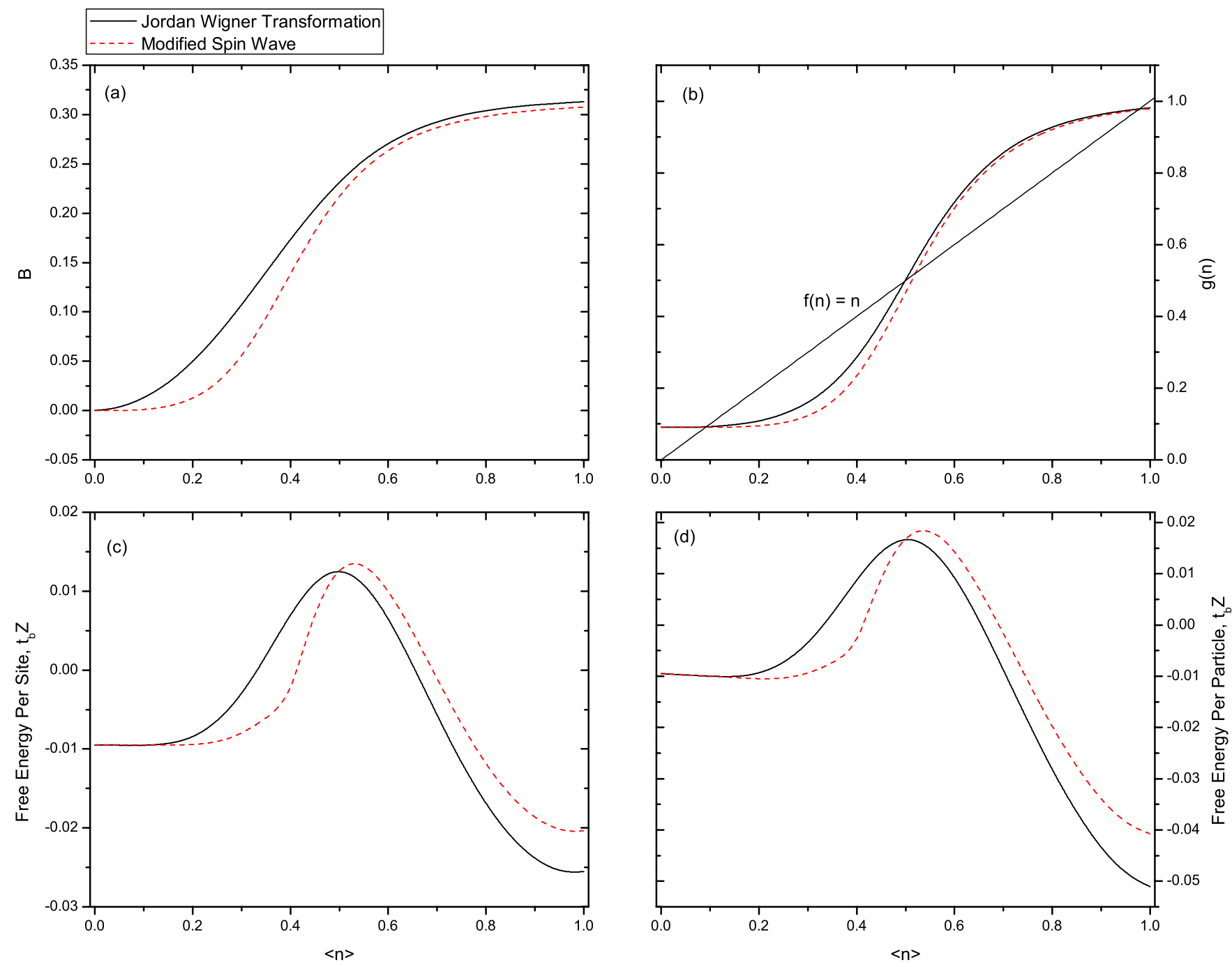

FIG. 7. (Color online) 1D MFT results using the modified spin-wave method and using Jordan-Wigner transformation. (a) Correlation function $B(\bar{n}, T)$, (b) the self-consistent solutions $\bar{n}$, (c) free energy per site, and (d) free energy per particle. System parameters are $\Delta / t_{b} Z=0.3$ and $k T / t_{b} Z=0.1$.

At finite $T$, the free energy due to $H_{s}$ can still be calculated exactly in one dimension, but the MFT procedure we use to decouple $\hat{S}$ and $\hat{n}$ is an approximation. The MF finitetemperature results for the $1 \mathrm{D}$ case by both the modified spin wave and Jordan-Wigner transform are shown in Figs. 7 and 8 . The two methods give similar predictions in the spin correlation and the self-consistent $n$ solutions. The results of free energy obtained by the two methods exhibit some difference, but the lower the temperature the smaller the discrepancy. More importantly, the reentrance behavior from defect poor to defect rich back to defect poor phases on the MI side appears in both methods. Both methods give phase transitions in $\bar{n}$ at finite temperatures in one dimension, which is of course incorrect and an artifact of MFT, and the transitions should be interpreted as crossovers in defect densities rather than real phase transitions. Nevertheless, the 1D results provide additional support for the 3D phase diagram presented above.

The results presented so far are relevant for bosons in optical lattice, where the experiments may be performed at constant chemical potential and the lattice constant is fixed by the optical lattice. To apply them to supersolid ${ }^{4} \mathrm{He}$, modifications are necessary for the following reason. In the case of solid ${ }^{4} \mathrm{He}$, the lattice constant is not fixed externally but self-determined. Instead it is the number of helium atoms that is fixed. Therefore, when comparing the free energy of the defect poor and defect rich phases, one should use the free energy per atom rather than the free energy per site, with $N=N_{a}+N_{b}+N_{v}$, where $N$ is the number of sites, $N_{a, b}$ are the number of sites occupied by helium atoms in $a$ and $b$ states, and $N_{v}$ the number of vacancies. The number of ${ }^{4} \mathrm{He}$ atoms $N_{\mathrm{He}}=N_{a}+N_{b}$. Minimizing the mean field free energy per atom instead of per site gives small quantitative corrections, but do not change the main results presented above. The phase diagram in that case is shown in Fig. 9. The transition temperature is changed, but the first-order transitions and reentrance remain.

In summary, we have shown that the MI-SF transition of a two-band boson Hubbard model can differ significantly from that of the one-band model. Instead of a continuous transition, the transition here is first order, both for the $T=0$ and 

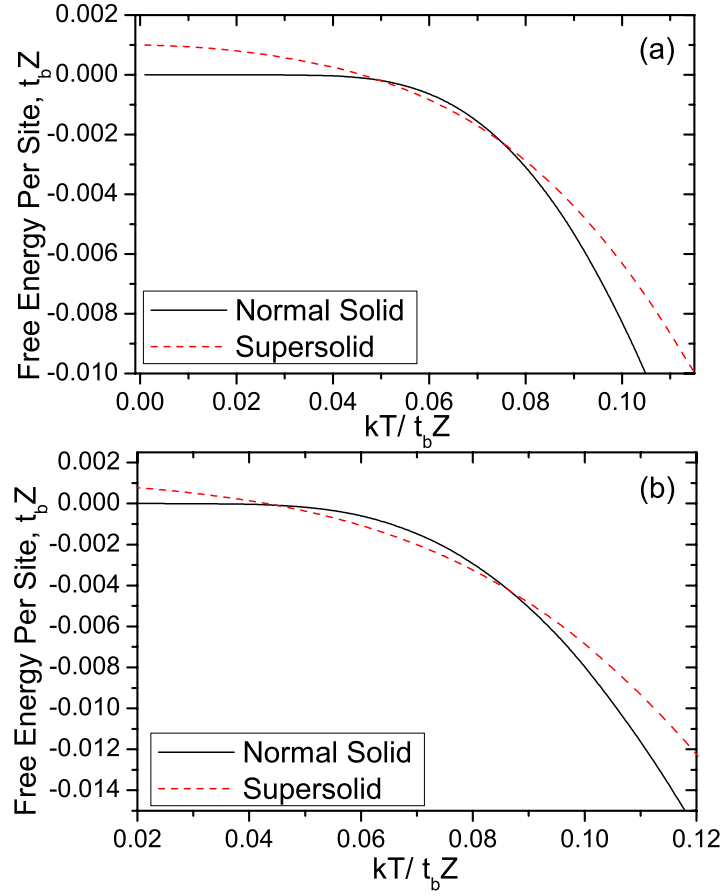

FIG. 8. (Color online) 1D MFT results of free energy per site of high $\bar{n}$ and low $\bar{n}$ self-consistent solutions indicating reentrance phase transitions by (a) modified spin-wave approximation at $\Delta / t_{b} Z=B_{1 D}^{*}+0.001$ and by (b) Jordan-Wigner transformation at $\Delta / t_{b} Z=B^{* *}+0.001$.

the finite $T$ transitions. On the MI side, there can be a reentrant transition into the SF phase and then back into the MI phase as temperature is raised. The underlying physics is that the MI-SF transition is actually a by-product of the transition between two competing phases, which are the defect poor and defect rich phases. In the defect poor phase, bosons are localized, while in the defect rich phase, they are delocalized and Bose condense. The defect rich phase contains a finite

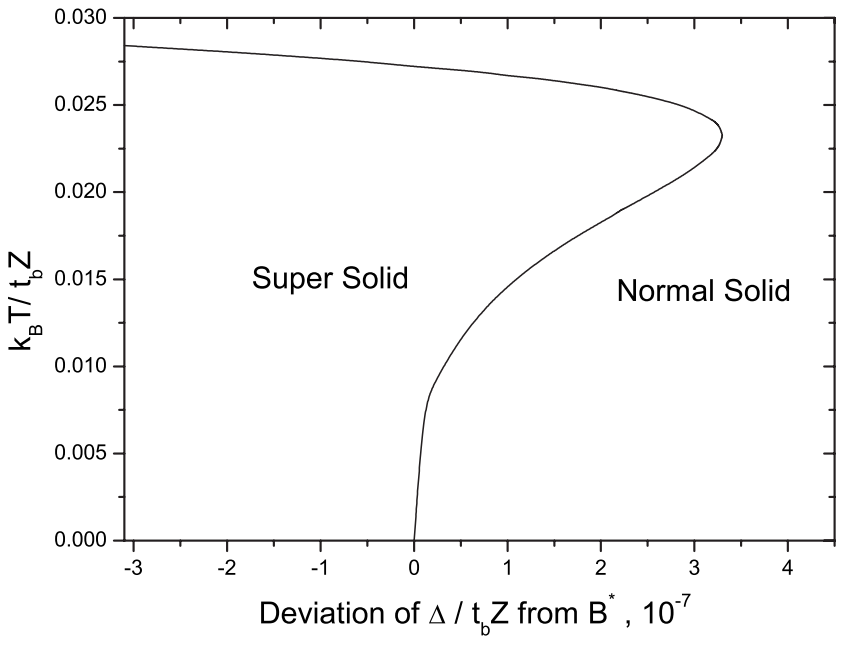

FIG. 9. Same as Fig. 6, but obtained by comparing free energy per particle rather than the value per site as shown in Fig. 6 .

density of excitons (defined in the Introduction) and vacancies even at $T=0$. Thus, the MI-SF transition is accompanied by a change in boson density in the case of optical lattice and a change from commensurate to incommensurate solid in the case of solid ${ }^{4} \mathrm{He}$. Our results confirm that the results obtained by DMZ are robust when phase fluctuations are included, and also extend their $T=0$ results to finite temperature. For three dimensions, these fluctuations are taken into account using a modified spin-wave theory. Although it is an approximate method, we believe it to be quantitatively reliable for our model by comparing the results in one dimension using this method and using Jordan-Wigner transformation.

\section{ACKNOWLEDGMENT}

This work was partly supported by the Hong Kong's RGC grant 706206.
${ }^{1}$ M. P. A. Fisher, P. B. Weichman, G. Grinstein, and D. S. Fisher, Phys. Rev. B 40, 546 (1989).

${ }^{2}$ E. Kim and M. H. W. Chan, Nature (London) 427, 225 (2004).

${ }^{3}$ E. Kim and M. H. W. Chan, Science 305, 1941 (2004).

${ }^{4}$ M. Greiner, O. Mandel, T. Esslinger, T. W. Hansch, and I. Bloch, Nature (London) 415, 6867 (2002).

${ }^{5}$ X. Dai, M. Ma, and F. C. Zhang, Phys. Rev. B 72, 132504 (2005).

${ }^{6}$ A. F. Andreev and I. M. Lifshitz, Sov. Phys. JETP 29, 1107 (1969)

${ }^{7}$ G. V. Chester, Phys. Rev. A 2, 256 (1970).

${ }^{8}$ A. J. Leggett, Phys. Rev. Lett. 25, 1543 (1970).

${ }^{9}$ Ann Sophie C. Rittner and J. D. Reppy, Phys. Rev. Lett. 97, 165301 (2006).

${ }^{10}$ M. Kondo, S. Takada, Y. Shibayama, and K. Shirahama, J. Low Temp. Phys. 148, 695 (2007).

${ }^{11}$ A. Penzev, Y. Yasuta, and M. Kubota, J. Low Temp. Phys. 148, 677 (2007).
${ }^{12}$ E. Burovski, E. Kozik, A. Kuklov, N. Prokof'ev, and B. Svistunov, Phys. Rev. Lett. 94, 165301 (2005).

${ }^{13}$ B. K. Clark and D. M. Ceperley, Phys. Rev. Lett. 96, 105302 (2006).

${ }^{14}$ J. Day and J. Beamish, Phys. Rev. Lett. 96, 105304 (2006).

${ }^{15}$ S. Sasaki, R. Ishiguro, F. Caupin, H. J. Maris, and S. Balibar, Science 313, 1098 (2006).

${ }^{16}$ L. Pollet, M. Boninsegni, A. B. Kuklov, N. V. Prokof'ev, B. V. Svistunov, and M. Troyer, Phys. Rev. Lett. 98, 135301 (2007).

${ }^{17}$ A. C. Clark, J. T. West, and M. H. W. Chan, Phys. Rev. Lett. 99, 135302 (2007).

${ }^{18}$ P. W. Anderson, W. F. Brinkman, and D. A. Huse, Science 310, 1164 (2005).

${ }^{19}$ B. A. Fraass, P. R. Granfors, and R. O. Simmons, Phys. Rev. B 39, 124 (1989).

${ }^{20}$ L. H. Nosanow and G. L. Shaw, Phys. Rev. 128, 546 (1962).

${ }^{21}$ M. Boninsegni, A. B. Kuklov, L. Pollet, N. V. Prokof'ev, B. V. Svistunov, and M. Troyer, Phys. Rev. Lett. 97, 080401 (2006). 
${ }^{22}$ D. E. Galli and L. Reatto, Phys. Rev. Lett. 96, 165301 (2006).

${ }^{23}$ M. A. Adams, J. Mayers, O. Kirichek, and R. B. E. Down, Phys. Rev. Lett. 98, 085301 (2007).

${ }^{24}$ D. Jaksch, C. Bruder, J. I. Cirac, C. W. Gardiner, and P. Zoller, Phys. Rev. Lett. 81, 3108 (1998).

${ }^{25}$ S. R. Clark and D. Jaksch, Phys. Rev. A 70, 043612 (2004).

${ }^{26}$ T. N. De Silva, M. Ma, and F. C. Zhang, Phys. Rev. B 66, 104417 (2002).

${ }^{27}$ G. Gomez-Santos and J. D. Joannopoulos, Phys. Rev. B 36, 8707 (1987).

${ }^{28}$ M. Takahashi, Phys. Rev. Lett. 58, 168 (1987).
${ }^{29}$ M. Takahashi, Phys. Rev. B 40, 2494 (1989).

${ }^{30}$ J. E. Hirsch and S. Tang, Phys. Rev. B 40, 4769 (1989).

${ }^{31}$ The finite-temperature first-order transition will terminate in a tricritical point and switch to second order as one moves sufficiently deep into the SF phase from the zero-temperature transition point. However, as this occurs within our model when the defect-free state, the vacancy, and the exciton are all nearly degenerate, it is outside the regime of interest for the two-band model of this work.

${ }^{32}$ M. Blume, Phys. Rev. 141, 517 (1966).

${ }^{33}$ H. W. Capel, Physica (Utrecht) 37, 423 (1967). 